\title{
Tatiana Samrowski: Matherätsel (nicht nur) für Begabte der Klassen 4 bis 6
}

\author{
Springer 2021, XII + 230 Seiten, ISBN: 978-3-662-64014-2, €24,99; \\ e-Book ISBN 978-3-662-64015-9, €19,99
}

\section{Karl-Hermann Neeb}

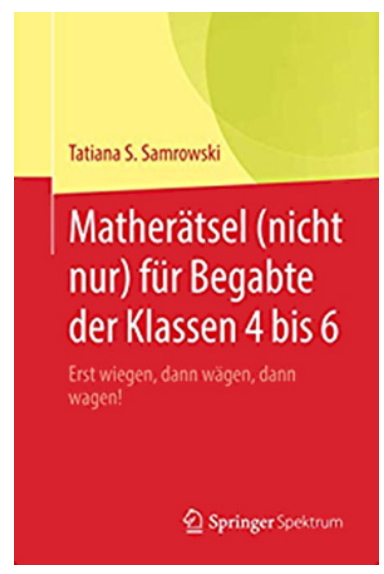

Das vorliegende Buch ist eine Sammlung von Aufgaben für Mathematik-interessierte Schüler der 4.-6. Klasse. Hierbei liegt das Ziel weniger auf einer frühen Förderung von mathematischen Begabungen, sondern vielmehr in der Bereitstellung von Aufgaben, die einerseits als (Rätsel-)zeitvertreib dienen können, andererseits aber auch sehr gezielt Methoden und Denkweisen einüben, die schrittweise an die Schulmathematik heranführen. Frau Samrowski möchte hiermit einen Kontrapunkt zu den zum Teil reizlosen mechanischen Rechenaufgaben setzen, die das Interesse an der Mathematik eher erlahmen lassen, als es zu beflügeln. Ich denke, das ist ihr sehr gut gelungen.

Entstanden ist das Buch aus einer Sammlung von Aufgaben, die für den Unterricht der Junior Euler Society für Mathematik der Universität Zürich der Klassenstufen 3/4 und 5/6 in den Jahren 2012-2020 verwendet wurden. Sie richten sich an eine Zielgruppe, die zwar an Mathematik interessiert ist, aber heterogen was Begabung und Wissensstand angeht. Die Aufgaben dienten insbesondere zur Vorbereitung der Teilnahme an mathematischen Wettbewerben. Das Buch richtet sich daher in erster Linie an interessierte Kinder und deren Eltern zur Vorbereitung auf Wettbewerbe und Aufnahmeprüfungen für höhere Schulen. Natürlich bietet der reiche Aufgabenfundus auch Lehrern Material zur Vorbereitung von Wettbewerben und Ergänzungen zu den üblichen Lehrbuchaufgaben.

Karl-Hermann Neeb ( $\square)$

Universität Erlangen, Erlangen, Deutschland

E-Mail: neeb@math.fau.de 
Strukturiert ist das Buch in zwölf Kapitel, die sich verschiedenen Themen widmen, wie z.B. Ziffern, Wiegen und Gewichte, Rückwärtslösen, Zeit, Abzählen von Möglichkeiten etc. Die einzelnen Kapitel haben alle die gleiche Struktur: Sie beginnen mit einführenden Aufgaben, deren vollständige Lösung vorgestellt wird. Danach gibt es Aufgaben zum selbstständigen Lösen und Wettbewerbsaufgaben sowie einige Aufgaben zur Wiederholung vorher behandelter Themen.

Die Aufgaben variieren in ihrem Schwierigkeitsgrad, was aber nicht heißt, dass die Wettbewerbsaufgaben durchweg die schwierigeren sind. Da die einzelnen Aufgaben eher kurz gehalten sind, haben sie nicht den abschreckenden Effekt vieler überlanger Textaufgaben, wie man sie oft in Schulbüchern findet. Sie haben durchweg einen klaren Fokus und sind mit den eingeübten Methoden, und manchmal etwas zusätzlichem Knobeln und Nachdenken, gut lösbar.

Eltern und Lehrern, die Kinder mit etwas weniger mechanischen Aufgaben an die Mathematik heranführen wollen, bietet diese Aufgabensammlung einen reichen Fundus, in dem für jeden Geschmack etwas zu finden sein sollte.

Funding Open Access funding enabled and organized by Projekt DEAL.

Open Access Dieser Artikel wird unter der Creative Commons Namensnennung 4.0 International Lizenz veröffentlicht, welche die Nutzung, Vervielfältigung, Bearbeitung, Verbreitung und Wiedergabe in jeglichem Medium und Format erlaubt, sofern Sie den/die ursprünglichen Autor(en) und die Quelle ordnungsgemäß nennen, einen Link zur Creative Commons Lizenz beifügen und angeben, ob Änderungen vorgenommen wurden.

Die in diesem Artikel enthaltenen Bilder und sonstiges Drittmaterial unterliegen ebenfalls der genannten Creative Commons Lizenz, sofern sich aus der Abbildungslegende nichts anderes ergibt. Sofern das betreffende Material nicht unter der genannten Creative Commons Lizenz steht und die betreffende Handlung nicht nach gesetzlichen Vorschriften erlaubt ist, ist für die oben aufgeführten Weiterverwendungen des Materials die Einwilligung des jeweiligen Rechteinhabers einzuholen.

Weitere Details zur Lizenz entnehmen Sie bitte der Lizenzinformation auf http://creativecommons.org/ licenses/by/4.0/deed.de. 